\title{
Reflexiones en torno de la crisis actual del Mercosur en su CONFIGURACIÓN COMO ACTOR REGIONAL
}

\section{REFLECTIONS ON THE CRISIS AND CURRENT SITUATION OF MERCOSUR IN ITS CONFIGURATION AS A REGIONAL ACTOR}

\author{
Catherine Ortiz Morales
}

\begin{abstract}
RESUMEN
Este artículo se propone plantear el debate sobre la cohesión regional del MERCOSUR a partir de un modelo y marco analítico formulado para reflexionar acerca de factores que, en una relación superpuesta e interconectada, propone el análisis sobre el liderazgo, la interdependencia económica, la convergencia política, la identidad y la institucionalidad en la configuración del MERCOSUR como actor regional ante la coyuntura actual desde un enfoque ecléctico. En este sentido se toman en consideración debates y aportes teóricos de las Relaciones Internacionales, particularidades del proceso del MERCOSUR en sí mismo, así como algunas reflexiones que han tenido lugar en la academia latinoamericana para sentar bases sobre las cuales repensar la integración regional en el caso de MERCOSUR. Como resultado ha tenido lugar la necesidad de cambios en los paradigmas los cuales, implícitamente, han determinado el debate académico y político sobre los factores de cohesión como "factores de éxito" con un carácter normativo y prescriptivo desde el referente europeo y desde una perspectiva racional de las Relaciones Internacionales que ha dejado de lado un análisis instrumental de la realidad para generar reinterpretaciones y reflexiones acerca del proyecto de MERCOSUR desde sus particularidades, realidad y hechos actuales. Como conclusión, el momento al que se asiste en MERCOSUR representa una crisis en la teoría y práctica que plantea el escenario de oportunidad de repensar factores de "éxito" para su cohesión regional y configuración como actor regional dadas las debilidades y fortalezas en sus particularidades de liderazgo, interdependencia económica, convergencia política, identidad e institucionalidad.
\end{abstract}

\section{PALABRAS CLAVE}

Liderazgo, interdependencia, convergencia, identidad, institucionalidad, MERCOSUR.

\section{ABSTRACT}

This article aims to raise the debate on the regional cohesion of MERCOSUR, a model and analytical framework proposed to reflect on factors that, in an overlapping and interconnected relationship, proposes the analysis of leadership, economic interdependence, political convergence, identity and institutionality in the configuration of MERCOSUR as a regional actor in the current situation from an eclectic approach. In this sense, debates and theoretical contributions of International Relations are taken into consideration, particularities of the MERCOSUR process itself, as well as some reflections that have taken place in the Latin American academy to lay the foundations on which to rethink regional integration in the case of MERCOSUR. As a result, there has been a need for changes in paradigms which, implicitly, have determined the academic and political debate on cohesion factors as success factors with a normative and prescriptive character from the European reference and from a rational perspective of International Relations that has left aside an instrumental analysis of reality to generate reinterpretations and reflections on the MERCOSUR project from its particularities, reality 
and current facts. In conclusion, the moment we are witnessing in MERCOSUR represents a crisis in theory and practice that poses the scenario of opportunity to rethink "success" factors for its regional cohesion and configuration as a regional actor given the weaknesses and strengths in its particularities of leadership, economic interdependence, political convergence, identity and institutionally.

\section{KEY WORDS}

Leadership, interdependence, convergence, identity, institutionally, MERCOSUR 


\section{INTRODUCCIÓN}

Dado los 30 años del Tratado de Asunción se ponen en el debate público los avances y retrocesos del proceso de integración regional del MERCOSUR, a lo cual se suma la coyuntura a la que se asiste enmarcada por el escenario político actual y los efectos de la pandemia que ha representado un desafío a los procesos de integración latinoamericana para una acción colectiva.

El carácter normativo y prescriptivo del modelo europeo en los estudios de integración regional latinoamericana ha propuesto como parte de la receta mágica, factores de "éxito" como el liderazgo -eje paymaster-, la interdependencia economía y la institucionalidad en términos de una supranacionalidad; marco de referencia en el cual las debilidades de los procesos de integración latinoamericanos se exacerban.

Sin embargo, pese a las debilidades existentes, avances y retrocesos del caso MERCOSUR y, en la coyuntura actual en la que se demandan ajustes, cambios, reformas y flexibilización a su régimen regional, un aspecto clave que ha llamado la atención en los estudios de integración pasa por la cohesión regional a partir de las particularidades de cada estudio de caso que demanda repensar la integración como el liderazgo, la interdependencia económica, la convergencia política, la identidad y la institucionalidad.

El presente artículo busca retomar el modelo de cohesión regional y sus factores "de éxito" resaltando aportes, debates y reflexiones que han tenido lugar recientemente en el análisis académico latinoamericano para repensarse la integración regional, en particular, y ser aplicados en la reflexión actual del caso del MERCOSUR. En ese sentido se propone un marco teórico y conceptual ecléctico a partir de los debates y aportes existentes con respecto al liderazgo, la interdependencia económica, la convergencia política, la identidad y la institucionalidad; factores que guardan una relación superpuesta e interconectada.

Como marco contextual, para el estudio de caso propuesto, es pertinente resaltar el punto de inflexión al que se asiste como actor político regional en un nuevo ciclo, dada la coyuntura que marca el agotamiento del modelo integracionista -político, económico e institucional- ante las demandas de ajustes, cambios, reformas y flexibilización de su proyecto político, dado el juego de intereses resultante de un reordenamiento de las relaciones económico-comerciales intra y extrarregionales. 


\section{MOdELO DE ANÁLISIS DE COHESIÓN REGIONAL PARA LA CONFIGURACIÓN DE UN ACTOR REGIONAL}

Partiendo de la premisa de las regiones como unidades de análisis a partir de las cuales se configuran como actores políticos regionales en el sistema internacional (Ortiz Morales, 2020), la integración regional latinoamericana presenta debilidades y fortalezas estructurales para responder a los cambiantes escenarios de oportunidades y amenazas existentes. Este es el caso de MERCOSUR que enfrenta un punto de inflexión en el escenario actual, tanto por (1) factores externos y sistémicos, así como, principalmente, (2) regionales e (3) internos (domésticos) que amenazan su cohesión regional por la falta de consenso ante el cambiante escenario político, dados los giros políticos e ideológicos que ha traído consigo los distintos ciclos electorales (Lorenzini y Pereyra, 2019).

Los referentes académicos en materia de integración regional han reconocido, de la mano de su multidimensionalidad, la complejidad del proceso (regionalización) y proyecto político (regionalismo), el cual ha sido institucionalizado en distintos tipos de multilateralismo regional (Mellado, 2018). De esta manera, para la Teoría del Nuevo Regionalismo se parte de la premisa sistémica de que una región pasa de ser un objeto pasivo a ser un sujeto activo dado sus niveles de regionalidad -regionalización- (Figura 1 a) como es (1) un espacio regional, (2) un complejo regional, (3) una sociedad regional, (4) una comunidad regional y (5) un sistema regional institucionalizado (Hettne, 2002; Hettne y Söderbaum, 2000).

Figura 1. Marco analítico: modelo de cohesión regional y factores de análisis para la configuración de un actore regional

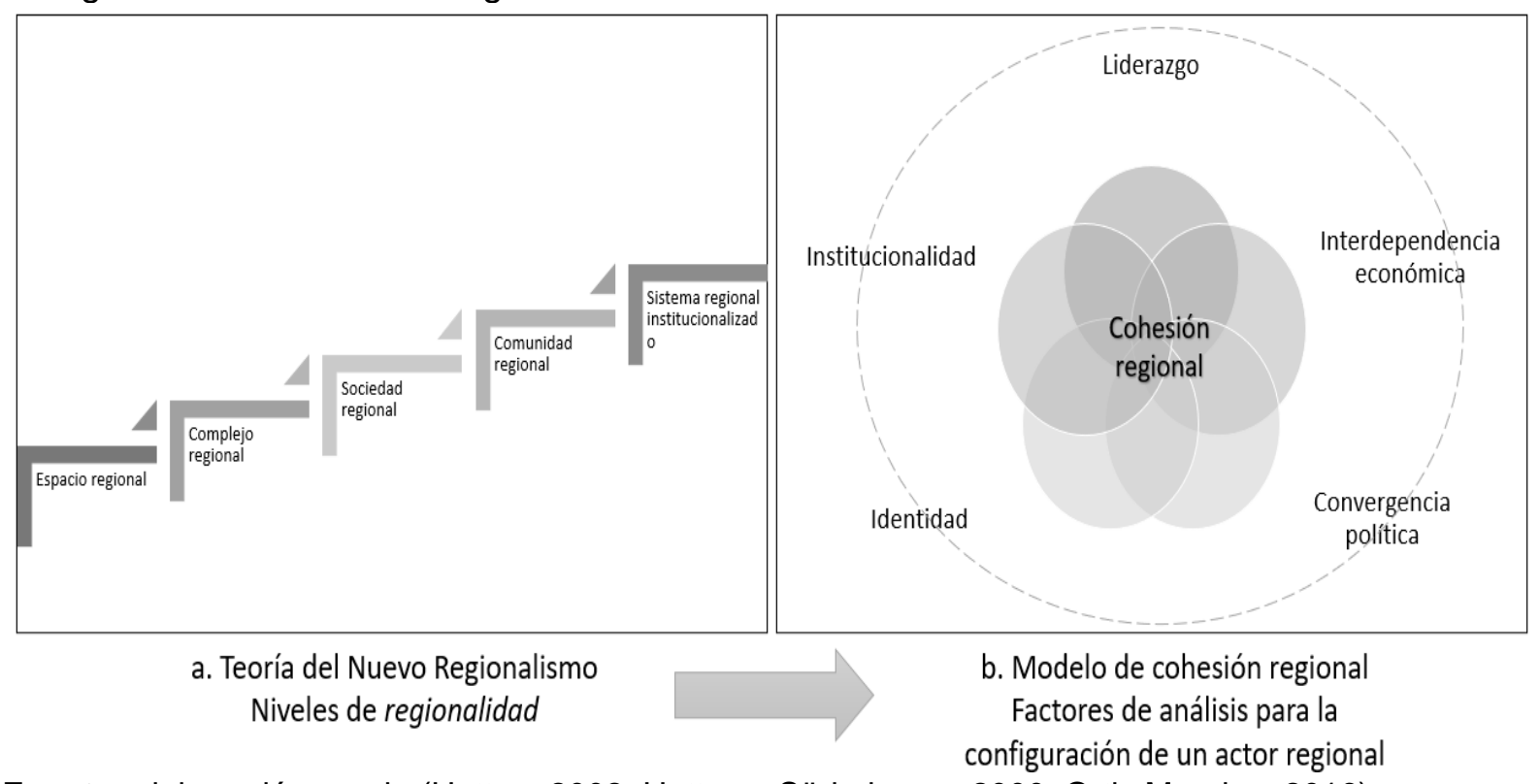

Fuente: elaboración propia (Hettne, 2002; Hettne y Söderbaum, 2000; Ortiz Morales, 2016). 
No obstante, más allá de una visión darwiniana, lineal, de evolución y progreso (Figura 1.a), como el mismo referente y visión de la teoría de integración económica regional de la escala de Balassa (1964), la teoría política Neo-funcionalista (spillover) (Hass, 2004) y la teoría del Nuevo Regionalismo (Hettne, 2002; Hettne y Söderbaum, 2000), para que un actor regional se configure como un sujeto activo se requiere, en distintos grados o niveles, de la cohesión regional resultante de factores como el liderazgo, la interdependencia económica, la convergencia política, la identidad regional y la institucionalidad como factores que guardan una relación superpuesta e interconectada (Figura 1. b) a partir de la interpretación y reflexión sobre las particularidades de cada proceso y proyecto.

Dichos factores y sus distintas perspectivas varían desde los diferentes referentes teóricos, en particular, de las Relaciones Internacionales -racionalismo, reflectivismo, aportes del sur global, entre otros- lo que ha da paso a abordajes eclécticos (Ortiz Morales, 2016); un escenario de oportunidad académica tanto para explicar (causalidad) pero, más importante aún, para interpretar y reflexionar acerca de los procesos de integración a partir de una lógica y razonamiento complejo de factores de cohesión regional que guardan una relación superpuesta e interconectada en la configuración de un actor regional.

En primera instancia, se toma como referencia un marco analítico propuesto (Figura 1.b) para la construcción de un modelo de cohesión regional que permita el análisis académico e instrumental del objeto de estudio a partir de categorías y factores abordados desde un referente ecléctico. En un primer momento, se toman como referencia, principalmente, enfoques racionalistas (realismo, interdependencia e institucionalismo) y de reflectivismo moderado (Constructivismo). Sin embargo, se resaltan discusiones y aportes de autores latinoamericanos que proponen repensar la integración latinoamericana para examinar y reflexionar las particularidades, así como las lecciones aprendidas en el caso concreto de MERCOSUR.

En ese sentido, sobre el liderazgo, una aproximación primigenia ha estado determinada por la perspectiva racionalista en el marco de la Teoría de Estabilidad Hegemónica. No obstante, autores latinoamericanos como Rocha y Morales (2018), proponen una aproximación al poder de los Estados tanto nacional como internacional desde, por una parte, la clásica concepción de poder a partir de las capacidades materiales o hard power que hace referencia al poder económico-militar (Realismo), así como a las capacidades inmateriales o soft power del poder político-comunicativo-cultural para la dirección y liderazgo político (Liberalismo). Sin embargo, se destaca como aporte académico la discusión de las capacidades semi-materiales que resalta el poder socio-institucional a partir 
de la fortaleza (o debilidad) para garantizar el bienestar, progreso y desarrollo con la legalidad en sus acciones, la provisión de servicios públicos y la prosperidad de sus ciudadanos a nivel doméstico.

De esta manera, en los estudios regionales, el liderazgo ha estado sustentado en la categoría de poder entendido, primordialmente, desde una concepción racionalista de poder material (económico-militar) así como inmaterial o soft power. Sin embargo, la característica principal del liderazgo entendido como estrategia, parte de ser cooperativa para la consecución de objetivos comunes (Destradi, 2010). Así, han tenido lugar aproximaciones desde la clásica Teoría de Estabilidad Hegemónica y el rol de paymaster que surge dada la posición relativa de actores claves los cuales se espera que asuman los costos de la integración y proporcionen bienes públicos regionales.

Como segunda categoría analítica se encuentra la interdependencia ${ }^{1}$. Es un concepto que, en las Relaciones Internacionales, surgió en la década de los 70 en el marco del debate interparadigmático desde el paradigma globalista, la teoría de la interdependencia y la teoría transnacional conocido como liberalismo duro (Frasson-Quenoz, 2015). Las cuatro características de la interdependencia en su conceptualización clásica, reconocen (1) la relación de dependencia mutua entre dos o más elementos o actores que (2) genera efectos recíprocos -costos y beneficios- así como (3) canales múltiples que conectan a las sociedades a nivel (i) interestatal -Estado-Estado-, (ii) transgubernamental -componentes de los Estados- y (iii) transnacional -actores extraestatales-, en donde es preferible (4) la cooperación dándose ganancias absolutas en una lógica de suma positiva. De esta manera se sustenta la premisa de que a mayor interdependencia no habrá uso de la fuerza y se reducen las posibilidades de conflicto (Keohane y Nye, 1988).

En este contexto, las discusiones actuales han buscado reconocer que los Estados no son el único actor entendido como actor central (estatocéntrico), racional (maximización de beneficios y reducción de costos) y unitario (división del mundo interno y externo, alta política y baja política). Por ende, dicha concepción clásica y racionalista que define el interés nacional desde una lógica de seguridad conlleva a sistemas de autoayuda que dan paso a una dinámica de dilema de seguridad y a un problema de acción colectiva (Wendt, 1992).

1 Desde el pensamiento latinoamericano se ha planteado el contrargumento a partir de la dependencia desde el paradigma estructuralista (Tickner, 2002). 
En línea con el liberalismo duro de las teorías de la interdependencia y transnacional, la generación de externalidades -efectos positivos y negativos- resultante, hace relevante la cooperación internacional que, según una de las definiciones canónicas, "[...] solo puede tener lugar en situaciones que contienen una mezcla de intereses contrapuestos y complementarios en donde los actores ajustan su comportamiento a las preferencias reales o previstas de los demás. La cooperación varía entre los temas y con el tiempo según las expectativas del futuro" (Axelrod y Keohane, 1985, p. 226).

Dado el anterior argumento teórico, es importante señalar dos aspectos. Por una parte, entre los objetivos de la cooperación internacional se encuentran gestionar la interdependencia (Bienes públicos mundiales o colectivos), promover normas y criterios sociales comunes (Bienes sociales universales) y reducir las desigualdades internacionales (cooperación para el desarrollo) (Ocampo, 2015). Así, puede ser entendido que la cooperación internacional, y no solo el rol de paymaster, permite proporcionar los bienes públicos en la integración repensando el liderazgo más allá de la posición jerárquica relativa a la estructura de poder regional de la Teoría de Estabilidad Hegemónica y basado en la racionalidad del eje ontológico individualismo -materialismo que ha dominado la agenda de investigación en el marco de relaciones dialécticas de conflicto-cooperación, interno-externo, amigo-enemigo, guerra-paz sobre la base del supuesto central del Estado como actor central, unitario y racional.

Por tanto, otros referentes como los aportes del sur global han buscado hacer énfasis en la relacionalidad basada en un eje ontológico holismo-idealismo (Tabla 1), dinámica en la que se hace referencia a las relaciones de ayuda mutua -obligaciones recíprocas- o relación de gana-gana, resaltando su influencia en el éxito económico que, como evidencia empírica, se ve reflejado en la interdependencia económica resultante del comercio intrarregional y la configuración de un orden regional sin importar los valores mesiánicos e individualistas (Creutzfeldt, 2011, 2012) sobre los cuales se sustenta el proyecto neoliberal (globalismo), su proceso actual de globalización, el individualismo metodológico (pesimismo antropológico) y las teorías racionalistas como la Teoría de Estabilidad Hegemónica en el nivel de la configuración del orden regional. 
Tabla 1. El debate onto-epistémico en las Relaciones Internacionales y la configuración de la hegemonía y el liderazgo

\begin{tabular}{|c|c|c|}
\hline \multirow[b]{2}{*}{$\begin{array}{l}\text { Relación agente- } \\
\text { estructura }\end{array}$} & Individualismo & Holismo \\
\hline & $\begin{array}{l}\begin{array}{l}\text { Explicaciones científicas (causalidad) } \\
\text { sobre las propiedades e } \\
\text { interacciones de los individuos. }\end{array} \\
\text { Las estructuras son secundarias en } \\
\text { el entendido que solo tiene efectos } \\
\text { en el comportamiento. }\end{array}$ & $\begin{array}{l}\text { Efectos de la estructura (ideas) } \\
\text { contribuyen a la construcción de los } \\
\text { agentes de manera co-constitutiva y sus } \\
\text { propiedades. } \\
\text { "El todo" constituye las identidades } \\
\text { e intereses de los Estados. }\end{array}$ \\
\hline \multirow[b]{2}{*}{$\begin{array}{c}\text { ¿De qué está } \\
\text { hecho el mundo? }\end{array}$} & Materialismo & Idealismo \\
\hline & $\begin{array}{l}\text { La importancia del poder e intereses } \\
\text { (exógenos) dada la organización } \\
\text { de fuerzas materiales. } \\
\text { Principio de racionalidad hacia la } \\
\text { maximización de los efectos positivos } \\
\text { (ganancias/beneficios) y la } \\
\text { disminución de los costos (efectos } \\
\text { negativos) de acuerdo con el interés } \\
\text { (realistas) o preferencias nacionales } \\
\text { (liberales). }\end{array}$ & $\begin{array}{l}\text { La importancia de las ideas como } \\
\text { significados intersubjetivos con efectos } \\
\text { constitutivos que configuran las } \\
\text { identidades y estos a su vez, los } \\
\text { intereses de manera endógena en el } \\
\text { proceso. } \\
\text { La naturaleza y la estructura de la } \\
\text { conciencia social sobre la base de } \\
\text { principios, normas e instituciones. } \\
\text { La estructura del sistema internacional } \\
\text { está compuesta primordialmente por } \\
\text { ideas. }\end{array}$ \\
\hline $\begin{array}{c}\text { Tipo de } \\
\text { comportamiento }\end{array}$ & $\begin{array}{l}\text { Racionalidad } \\
\text { HEGEMONIA }\end{array}$ & $\begin{array}{c}\text { Relacional } \\
\text { LIDERAZGO }\end{array}$ \\
\hline
\end{tabular}

Fuente: elaboración propia a partir de (Sodupe, 2019; Wendt, 2003).

Por otra parte, entendiéndose que la integración regional es un fenómeno multidimensional y complejo, cuyo alcance en la dimensión cooperativa de las relaciones internacionales puede ser entendida como el resultado último de la concertación y la cooperación de acuerdo con los componentes políticos de la integración (Tokatlian, 1994), la convergencia política es un tercer factor clave. Para Gardini (2010) la convergencia regional emerge como resultado del consenso respecto a la relación con la potencia hemisférica, el papel del líder regional y el modelo económico y de desarrollo; condicionantes inscritos en las políticas exteriores de los países latinoamericanos como asuntos de alta política que ha marcado la concepción y división del mundo interno y externo reduciendo, en muchos casos, el debate público de temas de alta política que obstaculizan la politización de la agenda y su democratización al ampliar el espacio político de participación más allá de una elite.

Se ha identificado que, en la región latinoamericana, las preferencias de política exterior resaltan que la ideología presidencial es la que más importa, por cuanto permite explicar el comportamiento de los Estados a partir de los cambios en la ideología del presidente y las características del ejecutivo, el contexto latinoamericano, el poder y el entorno institucional ante la ausencia de una (i) política exterior estable, a largo plazo y consensuada (Merke et al., 2020) o (ii) una política exterior pública basada en la relación Estado-sociedad que 
permita el vínculo de los condicionantes internos y la política exterior (interméstica) (Busso, 2019). Por tanto, una característica estructural latinoamericana es que la política exterior ha sido tradicionalmente de dominio exclusivo del ejecutivo y de una elite estrecha en la cual, más poder presidencial conduce a más cambios en la política exterior (Merke et al., 2020).

El cuarto factor hace referencia a la identidad. Frente a este aspecto, los constructivistas resaltan que la identidad y los intereses no son dados exógenamente, por lo que surgen preguntas sobre su formación siendo un proceso intersubjetivo en el que las identidades e intereses son endógenos a la interacción -relacional-. Sin embargo, la evolución de la cooperación permite transformar las identidades egoístas en colectivas.

De esta manera es importante el proceso (interacción y aprendizaje) y la influencia de la estructura (anarquía y distribución de poder) en el entendido que: "las transformaciones de identidad e interés a través del proceso son trasformaciones de estructura" (Wendt, 1992, p. 393). Por último, las ideas compartidas afectan las prácticas, identidades e intereses en cada momento histórico, así como la acción colectiva a partir de principios y normas compartidas afecta el comportamiento de los Estados (Wendt, 2003).

No obstante, respecto a la identidad, en los estudios de integración regional, en particular del caso latinoamericano, es importante resaltar dos aspectos. Por una parte, el debate sobre los valores e intereses. Por otra, la cultura integracionista. Para Gian Luca Gardini (2021) los valores e intereses son relevantes en los análisis de política exterior. Sin embargo, los valores compartidos han estado inscritos en las retoricas integracionistas que se sobrevaloran en el discurso. Por ende, lo que afecta la conducta política principalmente son los intereses los cuales se caracterizan por ser limitados. De esta manera, la realidad de los hechos en la región latinoamericana se ha visto constreñida en la práctica por altas expectativas, limitados intereses, valores compartidos sobrevalorados en el discurso en valores e intereses comunes y estratégicos sobre la supervivencia y la prosperidad (Gardini, 2021).

Por otra parte, Pablo Guadarrama (2021) resalta que la integración no depende solamente de la voluntad política de gobernantes, funcionarios o empresarios sino que se encuentra condicionada multifactorialmente. De esta manera, la cultura integracionista es un factor que la afecta y resalta que esta se entiende como "[...] ideas y acciones propiciadoras de un mayor grado de dignificación de los pueblos a través de su unión en aras de facilitar el intercambio reciproco de productos, de procesos materiales y espirituales [...]. Así, existen 
factores obstaculizadores y factores favorecedores en la cultura integracionista latinoamericana.

Por último, sobre la quinta categoría, aportes y debates sobre la institucionalidad como la argumentada y propuesta por Santos-Carrillo (2021) resalta que la realidad pasa por el análisis del diseño y las capacidades institucionales como marco analítico y su impacto en los problemas de acción colectiva dada la ineficacia institucional ante la ausencia de consensos respecto a los objetivos, la arquitectura institucional y la gobernanza de los distintos proyectos.

Este último aspecto, dadas las limitaciones en materia de participación y apertura de los procesos políticos hacia la ciudadanía puesto que predomina el papel de los gobiernos y, en ocasiones, los grupos empresariales socavándose la participación de la sociedad civil en las políticas regionales. De esta manera, el diseño de acuerdo con el tratado constitutivo, junto con otros factores, determina las capacidades entendiéndose en términos de la materialización de proyectos regionales, el cumplimiento de compromisos adquiridos así como los resultados y efectos de las organizaciones en políticas comunes o compartidas para alcanzar sus objetivos (Santos-Carrillo, 2019, 2021).

\section{Estado ACtUAL, DEBILIDADES Y FORTALEZAS DEL MERCOSUR: REPENSANDO LA COHESIÓN REGIONAL COMO ACTOR REGIONAL}

\section{a. Liderazgo: hacia una nueva discusión de tipos y estilos}

Una de las grandes cuestiones en el MERCOSUR ha sido el rol y posición de un líder regional desde la perspectiva de la teoría de Estabilidad Hegemónica y el denominado rol de paymaster. Dadas las capacidades que sobresalen a simple vista (Figura. 2), en múltiples ocasiones se ha asistido a la ausencia de pretensión de ejercer dicho rol. Por lo anterior, ha sido demandado un claro liderazgo para ser ejercido por Brasil y Argentina por su poder nacional e internacional en el tablero geopolítico del MERCOSUR desde sus antecedentes en el Acuerdo de Países de la Cuenca del Plata, la alianza político-diplomática ABC y el Protocolo de Integración Argentino-Brasileño (PICAB), entre otros, materializado e institucionalizado con el Tratado de Asunción.

El auge de las capacidades y poder de los miembros de MERCOSUR, con disparidades, se vio catapultado por el ciclo de los commodities que sustentó su modelo de integración revisionista en el marco de la ola de regionalismo poshegemónico y/o posliberal (20032016). Sin embargo, dado el fin del ciclo de los commodities y los cambios con los ciclos 
electorales en 2015, se inició un repliegue (Figura 2) que, a hoy, demanda ajustes, cambios y reformas al MERCOSUR. 
Figura 2. Índice de poder mundial, capacidades materiales, semi-materiales e inmateriales miembros de MERCOSUR 2003-2017

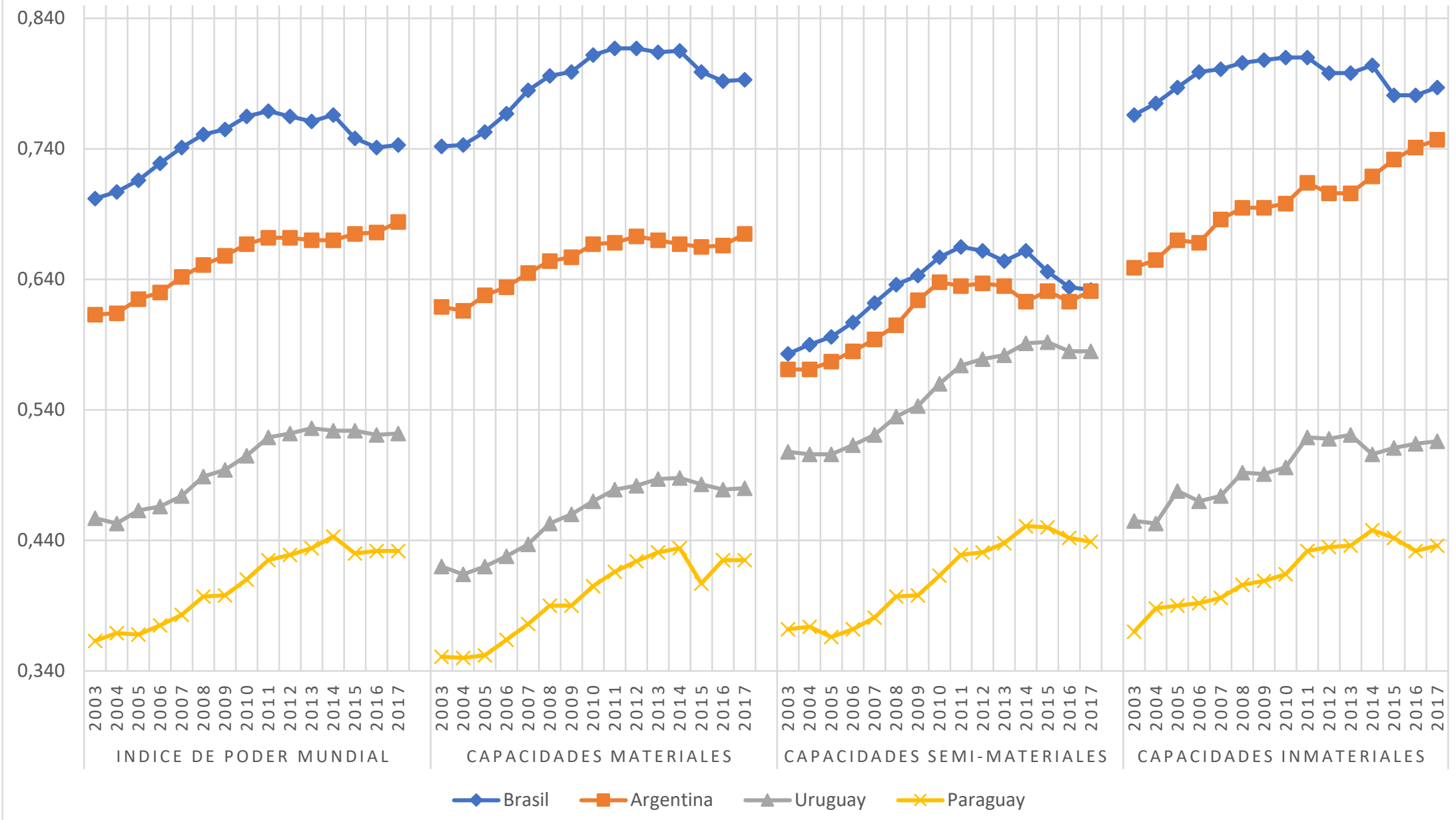

Fuente: elaboración propia a partir de (Morales Ruvalcaba, 2019). 
Desde una perspectiva alternativa para un liderazgo relacional, la principal debilidad estructural pero convergente de sus miembros se encuentra centrada en sus capacidades semi-materiales: poder socio-institucional a partir de la fortaleza o, principal debilidad (Figura. 1), para garantizar el bienestar, progreso y desarrollo con la legalidad en sus acciones, la provisión de servicios públicos y la prosperidad de sus ciudadanos a nivel doméstico de acuerdo con la riqueza y bienestar, la producción per cápita; la población; el consumo (gasto de consumo final de los hogares per cápita); la energía, la educación; y, la salud (Morales Ruvalcaba, 2019).

Lo anterior evidencia que, uno de los principales obstáculos en el MERCOSUR para un liderazgo regional al estilo de la Teoría de Estabilidad Hegemónica, se encuentra en sus problemas domésticos. Sin embargo, paradójicamente representa un escenario de oportunidad ante la razón de ser de la integración (Briceño, 2018), la cooperación internacional, las políticas regionales y sectoriales, y la coordinación y armonización de políticas públicas para una integración positiva resultante de una lógica relacional (Tabla 1) que, desde allí, permita repensar el factor de liderazgo en el debate regional.

\section{b. Interdependencia económica: ¿un falso dilema en el escenario actual?}

En cuanto a la interdependencia, las disparidades económicas también se hacen visibles pese a que sus intercambios comerciales han generado lazos de interdependencia económica en comparación a otros procesos regionales. Sin embargo, la coyuntura actual se ha centrado en el arancel externo común y la flexibilización para negociar acuerdos comerciales en forma individual puesto que los compromisos adquiridos para la construcción de una Unión Aduanera y la constitución de un mercado común establecido desde su Tratado de Asunción en $1991^{2}$ tienen como implicancia la liberalización de bienes, servicios y factores productivos; la eliminación de derechos aduanero y otras restricciones no arancelarias; un arancel externo común; la adopción de una política comercial común; la coordinación de posiciones en distintos foros económico-comerciales; la coordinación de políticas macroeconómicas y sectoriales; el compromiso de armonizar

2 De acuerdo con el Artículo 8 de Tratado de Asunción (1991) respecto a negociaciones comerciales externas que requerirán, en adelante, la coordinación de posiciones en la negociación de acuerdos frente a terceros siendo una función del Grupo Mercado Común como órgano ejecutivo (artículo 13). 
sus legislaciones para lograr el fortalecimiento del MERCOSUR, entre otros (Tratado de Asunción, 1991).

Empero, no se ha logrado materializar su unión aduanera perfecta ni eliminar las asimetrías internas, porque las posibilidades de convergencia han enfrentado importantes diferencias sectoriales (Figura 3) que, sumado a los diferentes grados y niveles de desarrollo industrial, conllevó a divergencias en las motivaciones y a diferencias de intereses comerciales que han buscado ser sorteadas mediante instancias de alto nivel político dada la configuración de un AEC, en particular, de bienes para la modernización mediante el acceso de la maquinaria y equipos necesarios (bienes de capital); diferencia del gigante, Brasil, quien contaba con una industria establecida.

Figura 3. Desglose de las exportaciones e importaciones totales de la economía (2018)

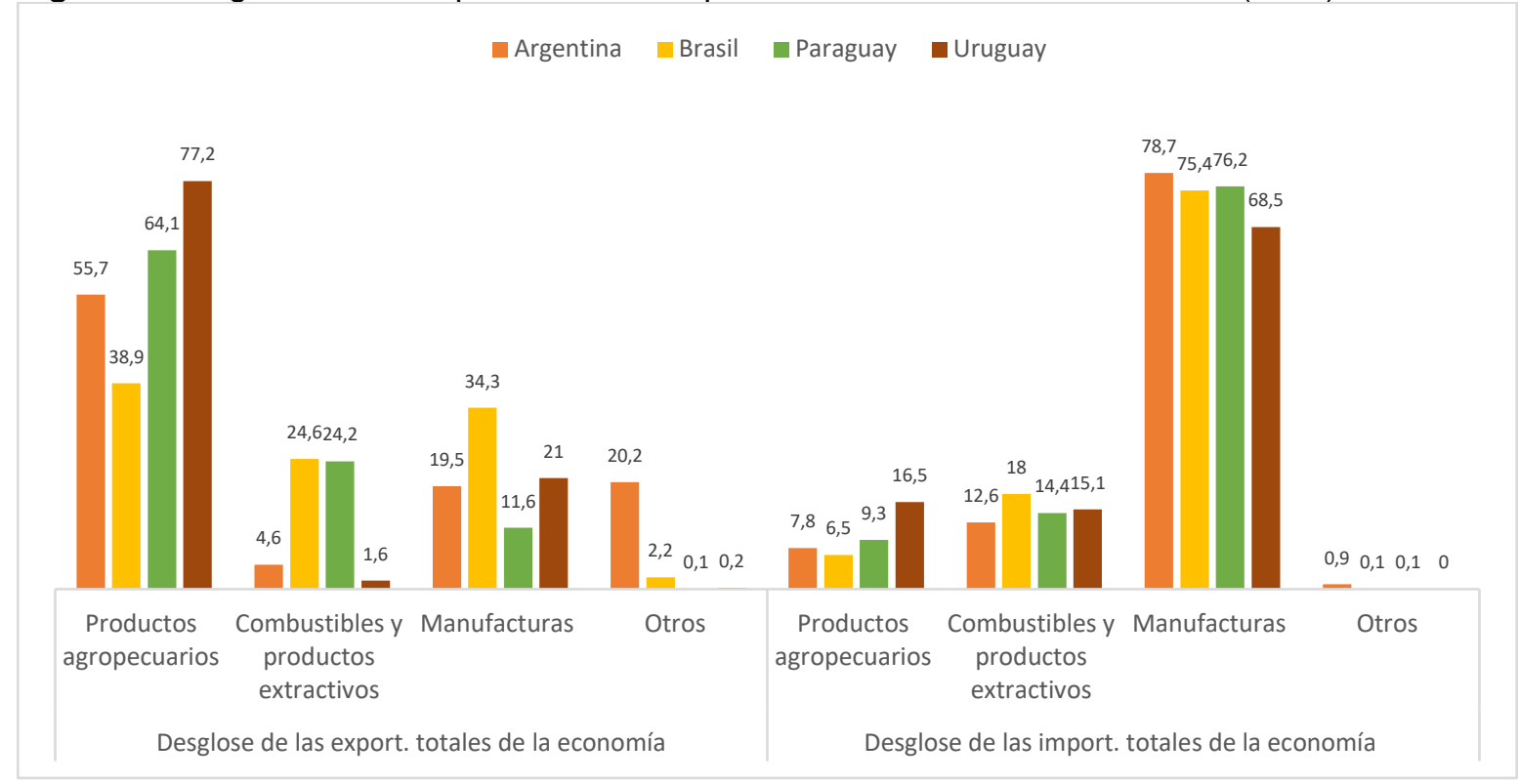

Fuente: elaboración propia con datos de (OMC, 2020).

Actualmente, uno de sus principales debates se centra en la evolución del proyecto MERCOSUR en cuanto a su relacionamiento externo dada la consolidación, flexibilización o eliminación de la Unión Aduanera que daría paso a una variación en la evolución, por su trayectoria y proceso en fits and starts, rompiéndose así una lógica neofuncionalista de autosostenible (spillover) de acuerdo con las teorías políticas tradicionales de integración regional (Moravcsik, 1993). A partir de la teoría de integración económica, lo anterior daría paso a su reformulación en una zona de libre comercio. 
Sin embargo, una de las principales amenazas actuales es la creciente incertidumbre, a lo que se ha sumado la tendencia de desaceleración, en parte como consecuencia de los efectos económicos de la pandemia que, estructuralmente, le antecedieron el desgaste del modelo neoliberal (globalismo) y sus dos pilares, libre mercado/comercio y democracia liberal, que sustentó el proceso globalizador en un escenario de tensiones comerciales que, a nivel regional, se suma el fin del ciclo de los precios de los commodities.

Figura 4. Comercio intrarregional total de bienes

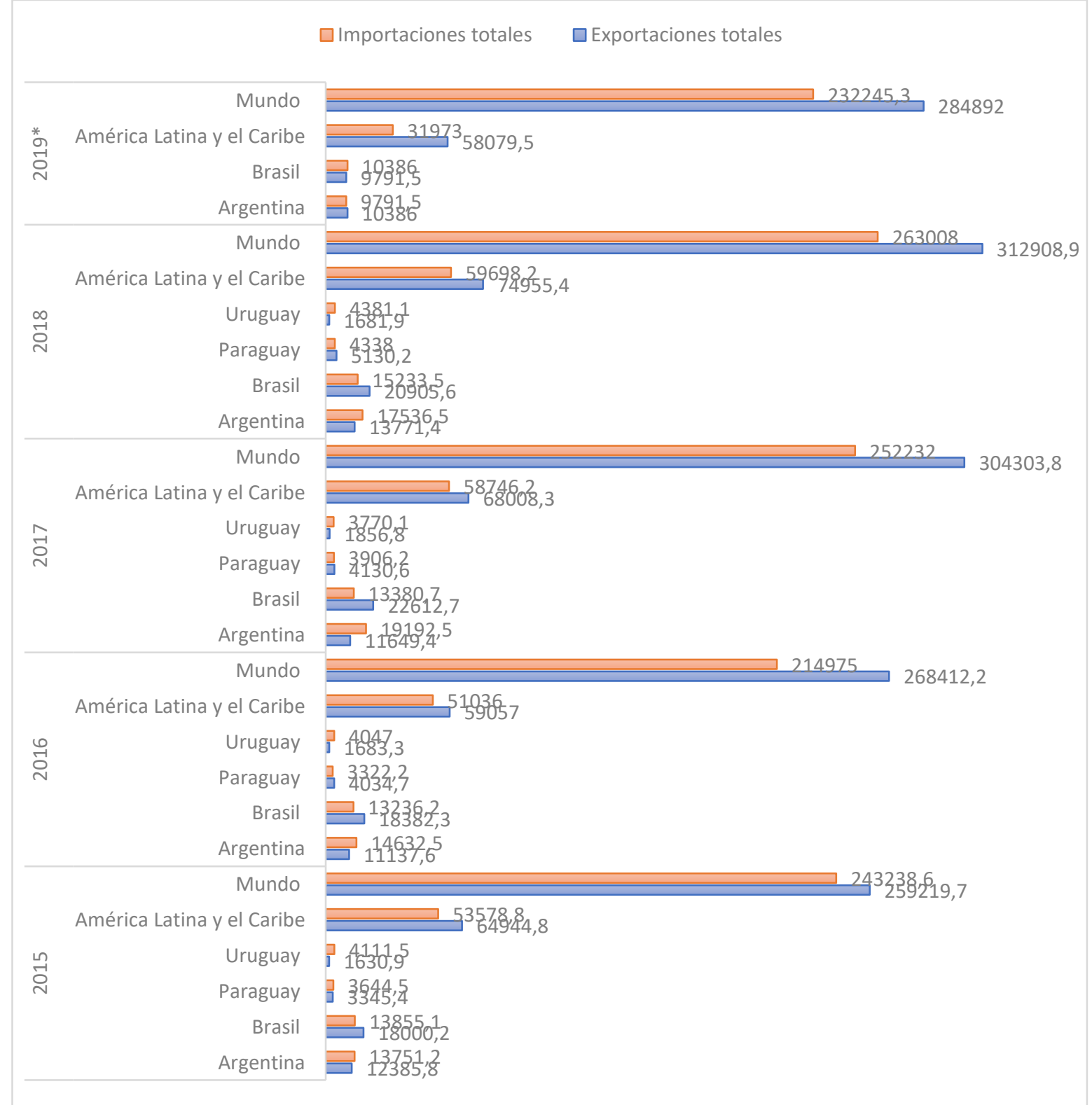


Nota: Datos expresados en millones de dólares. Los datos de 2019 solo incluyen el comercio bilateral de Argentina y Brasil y el comercio de estos dos actores con América Latina y el Caribe, así como con el mundo. Los datos de las exportaciones e importaciones de América Latina y el Caribe, así como del mundo, son los intercambios de los cuatro países miembros. Elaboración propia con datos de (CEPALSTAT, 2021).

Otro aspecto que ha caracterizado el punto de inflexión ha sido el panorama macroeconómico con déficits fiscales y externos de sus miembros dado el deterioro en 2018, "[...] debido a un contexto internacional más adverso y a factores internos. Con un bajo crecimiento de Brasil y un menor dinamismo relativo de Paraguay y Uruguay, el mayor cambio se observó en Argentina, que entró en recesión" (Rozemberg et al., 2019, p. 10).

A lo anterior se suma que el comercio de bienes y servicios del MERCOSUR se ha visto impulsado por las importaciones de Brasil y Argentina (Figura 4) y las exportaciones de sus miembros, principalmente Brasil, a socios de la extrazona (Figura 5). Así, la demanda y presencia de China como la importación de sus productos en un escenario en el que, a la par, se ha asistido a un descenso en la participación de los países socios del bloque regional reflejado en una disminución de las exportaciones intrazona (Figura 5). De esta manera, se asiste a déficits comerciales principalmente para Argentina y Uruguay, pero superávits como bloque regional en sus intercambios con América Latina y el Caribe y con el resto del mundo (Figura 4).

Figura 5. Principales destinos y orígenes del comercio de mercancías (2018-2019)

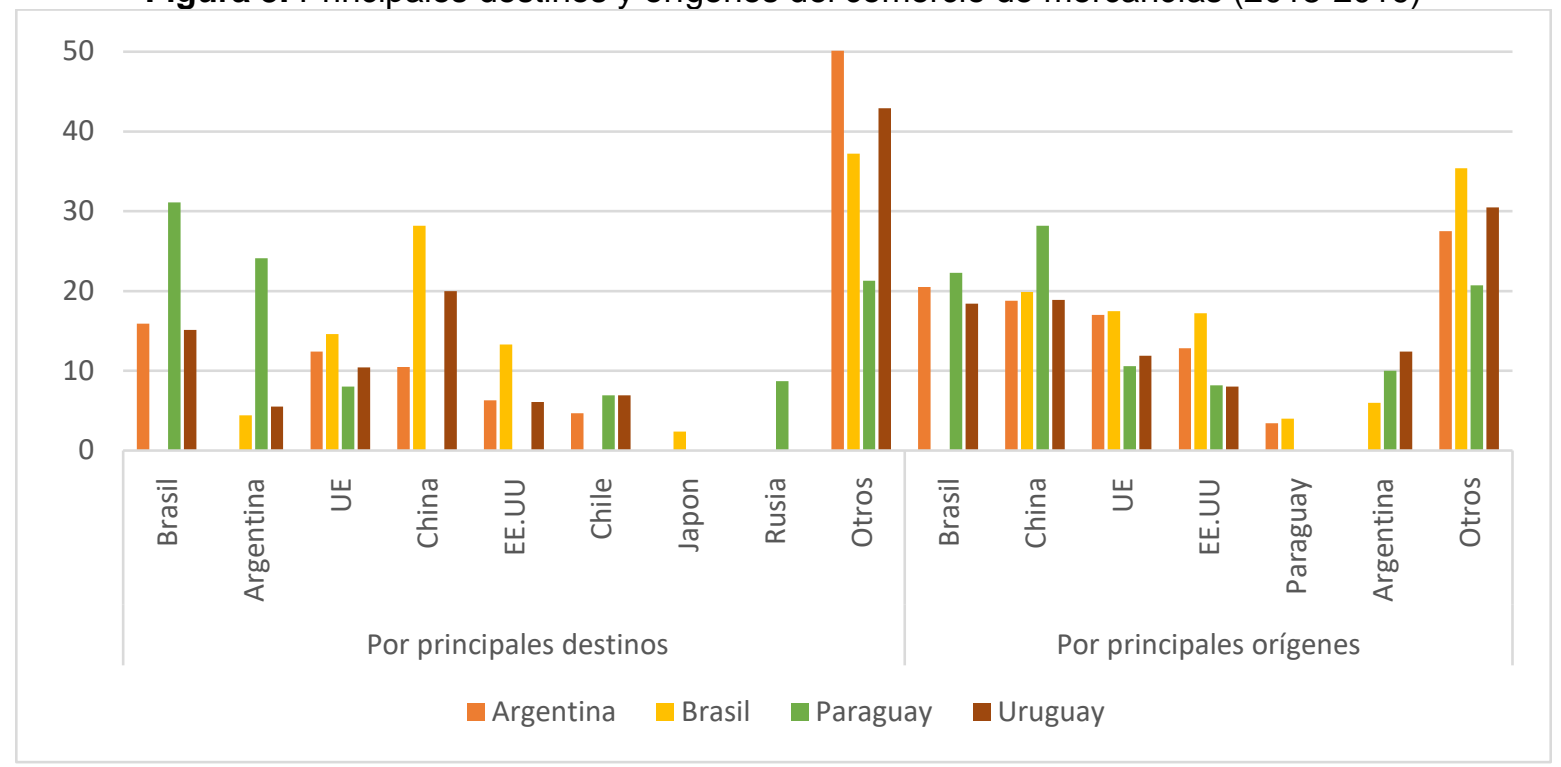

Fuente: elaboración propia con datos de (OMC, 2020). 
Esta realidad económica y como resultado de su grado de profundización económica en la construcción de una Unión Aduanera hacia un mercado común, ha dado paso a distintas visiones sobre la integración política. Actualmente, el tablero geopolítico y estratégico ha estado marcado, principalmente, por los intereses de la dupla Brasil y Uruguay cuya posición ha estado centrada en propender el crecimiento a través de la flexibilización del comercio intra y extra regional.

c. Convergencia política: de la centralización (intergubernamentalismo) de una política exterior hiperpresidencialista a la descentralización (gobernanza) de la integración regional.

La convergencia política ha estado determinada por las divergencias que han resultado de posiciones dialécticas y dicotómicas. Por una parte, Brasil defiende flexibilizar el MERCOSUR en aras de poder negociar acuerdos comerciales con otros países de manera bilateral. Para este propósito, la estrategia se centra en una reducción del arancel externo común. Por otra parte, la posición, principalmente de Argentina, orientada a la protección de la industria nacional ante el ingreso de productos importados con bajos costos que podría terminar de afectar las economías ya golpeadas por la actual situación.

Los cálculos racionales pasan, por un lado, a blindar el proceso gradual de la profundización y nivel de integración económica en el proceso del MERCOSUR y, por otro ,al clásico debate de creación y desviación del comercio puesto que han sido insuficientes los resultados a 30 años de la armonización de instrumentos de política comercial y coordinación de políticas macroeconómicas (Tratado de Asunción, 1991).

Lo anterior, en términos teóricos, puede representar una evidencia práctica que sustenta las posiciones políticas de los actores respecto al dilema de (i) la vinculación entre las partes en una unión aduanera con un arancel externo común y un mercado común con la liberalización de factores de producción, y (ii) la desviación de comercio que se puede generar como efecto negativo (Petit, 2014). Sin embargo, pueden diferir los costos y beneficios por la prevalencia de cualquiera de las dos decisiones. Lo cierto es que, para contra argumentar los efectos de este dilema, vale la pena señalar tres hechos.

El primero, tiene que ver con la lección aprendida con la crisis y estancamiento perpetuado de la CAN a partir la coyuntura crítica a la que asistió por la ausencia de un 
consenso sobre el modelo de integración en sus dimensiones políticas, económicas e institucionales que dieron paso a una crisis en cada una estas dimensiones, desencadenando factores como la reducción del comercio intrarregional, el estancamiento de una unión aduanera perfecta hacia un mercado común y la subordinación o exclusión de una dimensión social fuerte y central en la agenda de integración andina que deja de lado la transformación productiva con equidad. Lo anterior, se vio representado en hechos como la salida de Venezuela, el abandono del proyecto de un arancel externo común y la negociación bilateral con Estados Unidos y la UE que llevaron a perder poder de negociación (Chaves García y Ortiz Morales, 2021).

El segundo hecho tiene que ver con el actual debate sobre la crisis de la globalización y/o globalismo (Ávila, 2016) a consecuencia de la desconfianza generada por los efectos negativos del libre mercado y libre comercio desde una óptica de best way, así como de la democracia liberal (Rodrik, 2012), pilares de la globalización en crisis que han sustentado el auge de gobiernos iliberales en el siglo XXI. A lo anterior se suma el constante "estado" de fragmentación y un constante "estado" de incertidumbre (Halperin, 2013).

En este escenario, se ha dado paso a nuevas tendencias como el trans-regionalismo que no debe desconocer el contexto de desacompasamiento que se ha dado en la región Latinoamérica en dos sentidos. Primero, entre las políticas exteriores con la divergencia de intereses y valores opuestos. Segundo, entre las estrategias de orden internacional y regional con los cambios políticos que han traído, en muchos lugares, tendencias proteccionistas con la vía media de un trans regionalismo selectivo, a la carta o globalización selectiva (Caballero, 2019).

Por último, vale la pena mencionar lo establecido en el artículo 4 del Tratado de Asunción (1991) en el que se establece "En las relaciones con terceros países, los Estados Parte asegurarán condiciones equitativas de comercio. A tal efecto, aplicarán sus legislaciones nacionales para inhibir importaciones cuyos precios estén influenciados por subsidios, dumping o cualquier otra práctica desleal". En este sentido, las desviaciones de comercio pueden estar dadas, en muchas ocasiones, por prácticas desleales de comercio institucionalizadas a través de medidas disconformes (Halperin, 2013) y la securitización del comercio internacional que justifica la subvención de exportaciones agrícolas por parte de rule makers (Halperin, 2020). 


\section{d. Identidad versus intereses: ¿elemento cohesionador o divergente?}

La discusión sobre la identidad en los procesos de integración regional, resalta que la misma puede ser un factor cohesionador, así como excluyente, dada la intersubjetividad en el proceso endógeno de la relación del yo (autoconcepto/autoimagen) y el otro entendido en el relacionamiento entre agentes que da como resultado su construcción sobre la base de los valores o sistemas de creencias. Así, dentro del regionalismo como proyecto político dirigido por los Estados, se contemplan las ideas, identidades e ideologías (Söderbaum, 2003). Para el constructivismo sistémico de Alexander Wendt (2003), de la identidad se construyen los intereses dada su formación en el proceso endógeno, a diferencia de los racionalistas para los cuales son exógenos (poder, paz, bienestar, seguridad).

A nivel nacional, esta identidad se puede ver expresada en formaciones discursivas y prácticas sociales desde donde se forman sus interpretaciones de acuerdo, también, a las identidades y expectativas del otro. A nivel institucional, esta identidad se materializa en las políticas exteriores de sus miembros, en los principios ordenadores del tratado constitutivo, así como de otros instrumentos, a partir de los cuales, se define la autoimagen y la imagen como actor regional (Ortiz Morales, 2016).

Al respecto, se estableció como piedra angular y base de su razón de ser y, por ende, de su identidad en el MERCOSUR, la integración como condición fundamental para acelerar sus procesos de desarrollo económico con justicia social "[...] mediante el más eficaz aprovechamiento de los recursos disponibles, la preservación del medio ambiente, el mejoramiento de las interconexiones físicas, la coordinación de las políticas macroeconómicas y la complementación de los diferentes sectores de la economía, con base en los principios de gradualidad, flexibilidad y equilibrio" (Tratado de Asunción, 1991).

Sin embargo, como se mencionó, la identidad puede ser un elemento cohesionador o excluyente y divergente. Dadas las motivaciones integracionistas o no, en los estudios de política exterior, las ideas y los valores no representan una relación causal directa en el comportamiento de los Estados sino son los intereses los que pueden afectar la conducta política (Gardini, 2021). Esta es la coyuntura actual del MERCOSUR en la cual, en la etapa del regionalismo poshegemónico y posliberal (2003-2016), existió un relativo 
consenso sobre el modelo integracionista de carácter revisionista con un componente del clásico regionalismo estratégico (1991), un regionalismo social (2003) y un regionalismo productivo (2003), que configuraron, en el escenario integracionista latinoamericano, su imagen y autoimagen como un modelo hibrido de integración regional frente al modelo ortodoxo de regionalismo abierto (Briceño, 2013).

Por otra parte, en MERCOSUR, los valores sustentados en la integración como condición fundamental para acelerar sus procesos de desarrollo económico con justicia social, en ocasiones han sido divergentes o subordinados a los intereses de actores involucrados de turno, así como a la prioridades cambiantes, temáticas y geográficas de la política exterior y de la elite de turno; los intentos por una institucionalidad al estilo europeo no han sido, por un lado, suficientes para blindar el proceso de integración a los giros y cambios políticos y, por otra parte, se ha justificado y sustentado discursivamente la polarización de visiones dicotómicas que marcan el pensamiento occidental y que restringen la politización y democratización de la integración regional en la agenda y debate nacional.

\section{e. Institucionalidad: más allá de lo supranacional.}

Como último debate se ha planteado la discusión sobre el diseño y las capacidades institucionales (Santos-Carrillo, 2019). Respecto del primero, se encuentra la clásica discusión del intergubernamentalismo y supranacionalismo. El primero, denotado por una alta diplomacia presidencial y su régimen regional arancelario (Acuerdo Arancelario).

El debate latinoamericano sobre la institucionalidad ha estado inscrito sobre la supranacionalidad y el intergubernamentalismo de las organizaciones regionales como instituciones internacionales dado su lógica en procesos de toma de decisiones y cesión de soberanía y/o competencias. No obstante, para Fernández-Guillén (2021), a nivel nacional, el rol central se concentra en las burocracias nacionales en la dimensión del Estado; sin embargo, en el regionalismo, el proceso incluye factores y procesos en que convergen voluntariamente países que son independientes y soberanos con sistemas políticos y legislativos propios.

De esta manera, los mecanismos institucionales de gobernabilidad que pueden ser intergubernamentales o supranacionales en lógicas institucionales diferentes para tomar 
decisiones, adoptar normas y solucionar controversias, deben tener en cuenta las particularidades históricas, sociales y culturales que configuran su estructura de acuerdo con las motivaciones que dan paso a los objetivos constitutivos; de estos últimos derivan los mecanismos de funcionamiento para su dirección, delegar soberaníasupranacionalidad- o ejercer la soberanía (Fernández-Guillén, 2021).

Al respecto, nuevos esquemas analíticos para comprender las especificidades de procesos de integración no europeos proponen una descentralización decisoria que de paso a la democratización para limitar la posibilidad de cambios de nuevos gobiernos con visiones contrarias (Pasquiarelo Mariano y Goulart Menezes, 2019).

En este factor, se resalta que muchos de los aportes y reflexiones pasan más allá del debate de la supranacionalidad en el que, la institucionalidad no debe estar más allá de los Estados sino por debajo de los mismos, es decir, que involucre a la sociedad en la interrelación de fuerzas y actores -gobernanza multinivel- para poder responder a las realidades locales, regionales, nacionales e internacionales desde una integración de abajo hacia arriba.

Por lo anterior, se requiere la politización y la democratización de la integración regional en la agenda nacional como un bien público con la participación de todos los actoresEstado, sociedad y mercado- que, pese a los espacios dados y reconocidos en el marco del MERCOSUR en la dimensión social y productiva, no ha sido suficiente para sacar las discusiones del nivel intergubernamental y su carácter centralizado por algunas elites que permita transitar de una región formal a la configuración de una región real. De esta manera, más allá de la supranacionalidad, los debates actuales pasan por proponer una real gobernanza regional.

\section{CONCLUSIONES Y REFLEXIONES FINALES}

Dada la coyuntura actual del MERCOSUR que demanda implícitamente redefinir las prioridades del proyecto regional del MERCOSUR, más allá del debate del regionalismo posliberal o poshegemónico que marcó el periodo 2003-2016, en el debate actual y ante la demanda de ajustes, cambios y flexibilización, en el presente artículo se toma en consideración repensar factores y condicionantes para una integración "exitosa" como el 
liderazgo, la interdependencia económica, la convergencia política, la identidad y la institucionalidad. Varios de los argumentos pasan por un cambio de paradigma que parte del debate onto-epistémico que ha dominado las agendas de investigación, así como las acciones y decisiones políticas y las interacciones regionales para repensarse la etapa actual del MERCOSUR y sus discusiones.

Respecto al liderazgo, una categoría principal de las relaciones de poder y de dominación en las lecturas clásicas de las relaciones internacionales, más allá de su estilo y práctica, pasa por la realidad de los factores o condicionantes domésticos en las capacidades semi materiales; principal debilidad estructural para un liderazgo al estilo de la teoría de estabilidad hegemónica, pero punto convergente para los miembros del MERCOSUR y razón de ser de la integración latinoamericana.

Los debates sobre la interdependencia económica y el descenso de esta enfrentan, para algunos la amenaza y para otros la oportunidad de las relaciones económicas y comerciales extrazona que, más allá de los costos y beneficios de la racionalidad, atentan contra la relacionalidad como posible principio ordenador. De esta manera, sin consolidar un modelo u organización regional anacrónica, el debate pasa por las constantes divergencias más que convergencias políticas que han limitado la consolidación del proyecto regional dada la referencia normativa y prescriptiva del modelo europeo pero que, posiblemente, puede abrir el debate sobre el pragmatismo del asiático.

Sin embargo, la realidad particular en Latinoamérica pasa por la polarización en constantes posiciones dicotómicos e intereses contrapuestos de su paradigmas y visiones de mundo que ha fragmentado la integración latinoamericana en general. Lo anterior, a consecuencia de la división clásica de mundo interno y externo en el que, los asuntos de la alta política no son temas de politización siendo la integración subordinada a la agenda temática y geográfica de la política exterior y el hiperpresidencialismo de turno.

Por último, más allá de los debates de supranacionalidad e intergubernamentalismo, la verdadera institucionalidad pasa por el espacio político para la participación de los actores involucrados que configuran una gobernanza regional (Estado, mercado y sociedad). Sin la democratización y politización de la agenda de integración, el peso que se le da a los 
principios, ideas, valores e identidad solo tendrá lugar a nivel discursivo dado que en la práctica no ha actuado como un elemento cohesionador.

\section{REFERENCIAS BIBLIOGRÁFICAS}

Ávila, R. (2016, 9 de Octubre). Las razones que tienen en entredicho a la globalización. El Tiempo. https://www.eltiempo.com/economia/sectores/razones-que-tienen-en-entredichoa-la-globalizacion-42093

Axelrod, R., y Keohane, R. O. (1985, October). Achieving cooperation under anarchy: Strategies and institutions. World Politics, 38(1), 226-254. https://doi.org/10.2307/2010357

Balassa, B. (1964). Teoría de la integración económica. Uteha.

Briceño Ruiz, J. (2013). Ejes y modelos en la etapa actual de la integración económica regional en América Latina. Estudios Internacionales, 45(175), 9-39. https://revistaei.uchile.cl/index.php/REl/article/view/27352

Briceño, J. (2018). Las teorías de la integración regional: más allá del eurocentrismo (Colección Acontecer Mundial Tom. 8). Ediciones Universidad Cooperativa de Colombia.

Busso, A. (2019). El vínculo entre los condicionantes internos y la política exterior. Reflexiones sobre el caso argentino. Ciclos en la historia, la economía y la sociedad, (52), 3-32. https://ojs.econ.uba.ar/index.php/revistaCICLOS/article/view/1388

Caballero, S. (2019). Regionalismo latinoamericano, multilateralismo y transregionalismo: divergencias, retroalimentaciones y potencialidades. En C. Ortiz Morales \& E. Vieira Posada (Eds.), Nuevas propuestas de integración regional: tendencias y retos de transformación (pp. 23-44). Ediciones Universidad Cooperativa de Colombia.

Chaves García, C. (2017, Enero-Junio). Aproximación teórica y conceptual para el análisis de la Alianza del Pacífico. Desafíos, 30(1), 21-45. https://doi.org/https://doi.org/10.12804/revistas.urosario.edu.co/desafios/a.5775

Chaves García, C., y Ortiz Morales, C. (2021). Crisis y ajustes de la CAN ante la ola del regionalismo posliberal y poshegemónico en el siglo XXI: análisis de la coyuntura crítica y cambios institucionales (En prensa).

Comision Economica para America Latina y el Caribe. (2021). Comercio intrarregional total de bienes. CEPALSTAT. Bases de datos y publicaciones estadisticas.

Creutzfeldt, B. (2011, November 30). Theory talk \#45: Qin Yaqing on rules vs relations, drinking coffee and tea, and a Chinese approach to global governance. Theory Talks. http://www.theory-talks.org/2011/11/theory-talk-45.html

Creutzfeldt, B. (2012). Theory Talk \#51: Yan Xuetong on Chinese realism, the Tsinghua School of international relations, and the impossibility of harmony. Theory Talks. http://www.theory-talks.org/2012/11/theory-talk-51.html

Destradi, S. (2010, November $1^{\text {st }}$.). Regional powers and their strategies: empire, hegemony, and leadership. Review of International Studies, 36(4), 903-930. 
Fernández-Guillén, O. E. (2021). ¿Supranacionalidad o intergubernamentalismo? Los casos ASEAN y Mercosur. https://gridale.org/videos-gridale-segundo-congreso/

Frasson-Quenoz, F. (2015). Autores y teorías de relaciones internacionales: una cartografía. Universidad Externado de Colombia.

Gardini, G. L. (2010). Proyectos de integración regional sudamericana: hacia una teoría de convergencia regional. Relaciones Internacionales, (15), 11-31. https://revistas.uam.es/relacionesinternacionales/article/view/5047

Gardini, G. L. (2021, 28 de Junio). Alemania y la Unión Europea apuestan por América Latina. Diario Concepción. https://www.diarioconcepcion.cl/opinion/2019/06/28/la-unioneuropea-y-alemania-apuestan-por-america-latina.html

Guadarrama-González, P. (2021). Presupuestos para caracterizar la cultura integracionista latinoamericana [Ponencia]. II Congreso latinomamericano de integracion regional, Buenos Aires, Argentina. https://youtu.be/bJKWJ3T6Oos?t=2929

Halperin, M. (2013). El sistema económico internacional y los avatares en la construcción de un nuevo multilateralismo. En N. Mellado y J. C. Fernández Saca (Eds.), Problemáticas del regionalismo latinoamericano en los inicios del siglo XXI (pp. 27-35). Editorial Delgado. http://sedici.unlp.edu.ar/handle/10915/59817

Halperin, M. (2020, Junio). El COVID-19 como renovado detonador del acaparamiento y la depredación comercial: ¿el multilateralismo en coma? Informe Integrar, (122), 2-42. https://www.iil.jursoc.unlp.edu.ar/textos/informe/integrar122.pdf

Haas, E. B. (2004). The Uniting of Europe: Political, Social, and Economic Forces, 1950-

1957. Notre Dame, Indiana: University of Notre Dame Press

Hettne, B. (2002,). El nuevo regionalismo y el retorno a lo político. Comercio Exterior, 52(11), 954-965. http://revistas.bancomext.gob.mx/rce/magazines/5/2/hett1102.pdf

Hettne, B., \& Söderbaum, F. (2000). Theorising the rise of regionness. New Political Economy, 5(3), 457-473.

Keohane, R. O., \& Nye, J. S. (1988). Poder e interdependencia: la política mundial en transición. Grupo Editor Latinoamericano. http://www.url.edu.gt/PortalURL/Biblioteca/Contenido.aspx?o=5244\&s=49

Lorenzini, M. E., \& Pereyra Doval, G. (2019). Posneoliberalismo y después: el centro relativo del sistema político y el ascenso de la marea celeste en el espejo de la política exterior de Argentina y Chile. Revista de Ciencia Política, 39(3), 435-457. http://ojs.uc.cl/index.php/rcp/issue/view/580

Mellado, N. (2018, Abril-Junio). El marco multilateral como herramienta esencial de la integración de ALC en un sistema multipolar. Derecho y economía de la integración, (6), 73-92. https://global.juri-dileyc.com/el-marco-multilateral-como-herramienta-esencial-de-laintegracion-de-alc-en-un-sistema-multipolar-pdf/\#

Merke, F., Reynoso, D., y Schenoni, L. L. (2020, September 8). Foreign policy change in latin America: Exploring a middle-range concept. Latin American Research Review, 55(3), 


\section{3-429. https://doi.org/10.25222/larr.380}

Morales Ruvalcaba, D. (2019). Database World Power Index [Data set]. World Power Index. https://www.worldpowerindex.com/data-wpi/

Moravcsik, A. (1993.). Preferences and power in the European community: A Liberal intergovernmentalist approach. Journal of Common Market Studies, 31(4), 473-524.

Ocampo, J. A. (2015). La gobernanza económica y social y el sistema de las Naciones Unidas. En J. A. Ocampo (Ed.), Gobernanza global y desarrollo: Nuevos desafíos y prioridades de la cooperación internacional (pp. 31-70). Siglo Veintiuno Editores Argentina S.A. https://repositorio.cepal.org/handle/11362/38855

Organización Mundial de Comercio. (2020). Perfiles Comerciales. Perfiles Comerciales, 2006-2019. https://www.wto.org/spanish/res s/reser s/trade profiles s.htm

Ortiz Morales, J. C. (2016). Modelo de análisis y factores de éxito para la cohesión regional: ¿la Alianza del Pacífico como actor regional? [Ponencia]. VIII Congreso de Relaciones Internacionales, Buenos Aires, Argentina. http://ocs.congresos.unlp.edu.ar/index.php/CRRII/CRRII-VIII/paper/view/3483

Ortiz Morales, J. C. (2020). Integración regional: las regiones como actores y unidades de análisis en las Relaciones Internacionales. En F. Sánchez \& N. Liendo (Eds.), Manual de Ciencia política y Relaciones internacionales (pp. 185-205). Universidad Sergio Arboleda. https://repository.usergioarboleda.edu.co/bitstream/handle/11232/1460/Integración regional.pdf?sequence $=1 \& i s A l l o w e d=y$

Ortiz Morales, J. C. (2021). Pensamiento politico occidental y pensamiento politico asiatico: una comprension desde las relaciones internacionales y su impacto en la configuracion del orden regional y mundial (En prensa).

Pasquiarelo Mariano, K. L., y Goulart Menezes, R. (2019). Reflexões teóricas sobre a Integração regional: a construção de novos pressupostos. En C. Ortiz Morales \& E. Vieira Posada (Eds.), Nuevas propuestas de integración regional: tendencias y retos de transformación (pp. 165-196). Ediciones Universidad Cooperativa de Colombia. Colección GRIDALE.

Petit Primera, J. G. (2014, Enero-Junio). La teoría económica de la integración y sus principios fundamentales. Revista Venezolana de análisis de coyuntura, 20(1), 137-162. https://issuu.com/iiesrodolfoquintero/docs/revista venezolana de an lisis de d232b36 $\underline{\text { bee12c9/130 }}$

Rocha Valencia, A., y Morales Ruvalcaba, D. (2018). El poder nacional-internacional de los Estados. Una propuesta transestructural. Geopolítica(s). Revista de estudios sobre espacio y poder, 9(1), 137-169. https://revistas.ucm.es/index.php/GEOP/issue/view/3327

Rodrik, D. (2012). La paradoja de la globalización: Democracia y el futuro de la economía mundial. Antoni Boch.

Rozemberg, R., Campos, R., Gayá, R., Makuc, A., \& Svarzman, G. (2019, Junio). Informe Mercosur: hacía un cambio necesario. Banco Intermamericano de Desarrollo. https://publications.iadb.org/publications/spanish/document/Informe MERCOSUR tiemps 
de cambio es es.pdf

Santos-Carrillo, F. (2019). De integración a cooperación regional. Aprendizajes a partir del análisis de las políticas regionales de la integración centroamericana. En C. Ortiz Morales \& E. Vieira Posada (Eds.), Nuevas propuestas de integración regional: tendencias y retos de transformación (pp. 119-162). Ediciones Universidad Cooperativa de Colombia.

Santos-Carrillo, F. (2021). Borrador de propuesta para la creación de un observatorio sobre las capacidades institucionales de las organizaciones de la integración latinoamericana. II Congreso Latinoamericano de Integración Regional.

Söderbaum, F. (2003). Introduction: Theories of New Regionalism. In T. M. Shaw \& F. Söderbaum (Eds.), Theories of New regionalism (pp. 1-21). Palgrave Macmillan.

Sodupe Corcuera,. K. (2019). La teoría de las relaciones internacionales a comienzos del siglo XXI. Universidad del País Vasco.

Tickner, A. (2002). Los estudios internacionales en América Latina: ¿subordinacion intelectual o pensamiento emancipatorio? Universidad de los Andes, CESO, Departamento de Ciencia Política: Alfaomega Colombiana.

Tokatlian, J. (1994). Los componentes políticos de la integración. En J. Acosta Puertas (Comp.), Integración, desarrollo económico y competitividad (pp. 49-58). CRESET.

Tratado de Asunción. (1991). Tratado para la Constitución de un Mercado Común entre la República Argentina, la República Federativa del Brasil, la República del Paraguay y la República Oriental del Uruguay. Mercosur. https://www.parlamentomercosur.org/innovaportal/file/15511/1/tratado_de_asuncion.pdf

Wendt, A. (1992). Anarchy is what states make of it: the social construction of power politics. International Organization, 391-425. https://doi.org/https://www.jstor.org/stable/2706858

Wendt, A. (2003). Social theory of International politics. Cambridge University Press.

Ortiz Morales, Catherine: Especialista Nacional. Centro de Pensamiento Global (CEPG), Universidad Cooperativa de Colombia. Miembro del Grupo de Reflexión sobre integración y desarrollo en América Latina y Europa (GRIDALE).

E-mail: jenny.ortizm@ucc.edu.co. https://orcid.org/0000-0001-9563-3115
Fecha de recepción: 18/08/2021
Fecha de aceptación: 22/09/2021 